\title{
Avances de Investigación en Educación Matemática: Tres transiciones cualitativas en proceso
}

\author{
Núria Planas, Universitat Autònoma de Barcelona (España)
}

Avances de Investigación en Educación Matemática: Tres transiciones cualitativas en proceso

\section{Resumen}

Nuestra revista está viviendo tres transiciones conectadas en las direcciones de: (i) llegar a ser más internacional, (ii) llegar a ser más influyente y (iii) llegar a ser más formativa. Como mecanismo al servicio de la comunidad de investigación en educación matemática, AIEM es un proyecto de contribución al desarrollo de esta comunidad mediante acciones dirigidas a situarse e impactar en el estado del arte de mayor nivel - que es necesariamente internacional - y a apoyar a los investigadores para que sus artículos sean útiles a estos dos fines. Este editorial problematiza ciertas interpretaciones de 'internacional', 'influyente' y 'formativa' y apuesta por la priorización de significados científicos.

Palabras clave. Proyecto AIEM; criterios científicos; llegar a ser (más) internacional; llegar a ser (más) influyente; llegar a ser (más) formativa.

Advances of Research in Mathematics Education: Three qualitative transitions in process

\section{Abstract}

Our journal is experiencing three related transitions in the directions of: (i) becoming more international, (ii) becoming more influential, and (iii) becoming more formative. As a tool serving the community of mathematics education research, AIEM is a project responsible for contributing to the development of this community through actions aimed at being put and impacting on the highest level state-of-the-art - which is necessarily international -, and at supporting researchers so that their articles are useful to these two aims. In this editorial, certain interpretations of 'international', 'influential' and 'formative' are problematized and then unpacked to prioritize scientific meanings.

Keywords. AIEM project; scientific criteria; becoming (more) international; becoming (more) influential; becoming (more) formative.

\section{El proyecto AIEM}

En mayo de 2012, con la publicación del primer número de 'Avances de Investigación en Educación Matemática', cristalizó una primera fase del proyecto colectivo de tener una revista oficial de la Sociedad Española de Investigación en Educación Matemática, que viajara de nuestra comunidad al resto del mundo. No estamos, pues, en la ocasión conmemorativa de ninguno de los aniversarios habitualmente celebrados en el mundo de las revistas científicas. Tampoco estamos en un año de cambio de editora, ni de cambio de título u otras cuestiones similares. Estas son ocasiones que no pasan desapercibidas incluso sin anunciarse o celebrarse mediante editoriales específicas. No obstante, el proyecto de la revista vive una segunda fase igualmente decisiva que sí debe ponerse de relieve y que es propia de cualquier proyecto de realización del campo intelectual y de circulación de ideas y de conocimiento. Si con anterioridad a 2012 se discutieron y andamiaron las razones de que la Sociedad tuviera una revista científica, en 2019 debemos preguntarnos acerca de cuáles son las razones de su subsistencia y buena salud. En esta segunda fase, AIEM experimenta tres transiciones fuertemente conectadas entre ellas. Su logro tiene que ver, en parte, con la participación de la comunidad que la guía e impulsa y que ha ido configurando un impresionante árbol genealógico desde los inicios del proyecto hasta hoy. Son transiciones con varias cotas sucesivas en las direcciones de: 
- Llegar a ser más internacional

- Llegar a ser más influyente

- Llegar a ser más formativa

Para que AIEM llegue a ser más internacional, más influyente y más formativa, el trabajo que se requiere es sobre todo cualitativo, de compromiso con tres cualidades inherentes al crecimiento y desarrollo de cualquier comunidad de investigación. Como mecanismo al servicio de la comunidad de investigación en educación matemática, AIEM tiene la responsabilidad de contribuir al desarrollo de esta comunidad a través de la intensificación de acciones unitarias dirigidas a situarse e impactar en el estado del arte de mayor nivel - que es necesariamente internacional - y a dar apoyo a los investigadores-autores para que sus artículos sean útiles a ambos fines. Estas acciones científicas en particular conllevan acciones técnicas orientadas a ganar presencia en bases de datos y a obtener medidas e índices que no deben confundirse con el fondo de lo que realmente importa y que la Agenda para la Acción 2018-2022 publicada en 2017 por nuestra Sociedad recoge. En el Ámbito 1 de esta Agenda - 'Generar espacios para facilitar y promover la comunicación y discusión de investigaciones en educación matemática' -, se alude al compromiso cualitativo de convertir la revista en referencia científica a través de la publicación de trabajos de relevancia. Se trata en definitiva de que AIEM sea una revista leída y estudiada por su público potencial. Este es el mayor reconocimiento que se le puede hacer a una revista de investigación.

\section{Llegar a ser más internacional}

El trayecto de AIEM y del proyecto que representa acumula varios logros, algunos en proceso. El inicio del proceso de llegar a ser más internacional se relaciona con la publicación de artículos por autores con afiliaciones institucionales de distintas partes del mundo, pero también con la incorporación de editores asociados del continente americano y de asesores científicos de hasta cinco continentes. Además, el rastreo de citas de artículos publicados en AIEM lleva cada vez más tanto a revistas locales con dominio de la lengua castellana, como a revistas europeas e internacionales con dominio o exclusividad de la lengua inglesa. Estas relaciones, sin embargo, no son todavía de naturaleza científica y no ahondan en el significado cualitativo de ser una revista de investigación (más) internacional. Como concepto, 'ser internacional' es difícil de definir debido a los múltiples focos e intenciones posibles. De la anterior enumeración de relaciones, por ejemplo, se infiere el uso de la lengua inglesa y la filiación territorial diversa en el conjunto de autores y en el de editores asociados y asesores. Pues bien, ser (más) internacional es algo más complejo que el resultado de un sumatorio de países y de lenguas involucradas en la elaboración de una revista. Estos focos son limitados y poco amplios, no atienden a criterios científicos y debe problematizarse la propensión a pensarlos como únicos o a valorarlos en exceso.

En el contexto de una revista comprometida con el desarrollo de una comunidad de investigación, el significado de 'ser internacional' se inspira en el objetivo de publicar artículos con resultados empíricos y reflexiones teóricas que contribuyan al avance/progreso del estado del arte y que se fundamenten en lo que se sabe y en lo que falta por saber. Este es el significado con foco científico que mueve nuestra labor en el equipo editorial y desde la dirección de la revista en coordinación con el comité asesor. $\mathrm{Si}$ bien es cierto que mencionar este objetivo puede parecer una trivialidad, no lo es en absoluto. Las dinámicas, exigencias y lógicas del mercado académico a menudo tienen como consecuencia ritmos frenéticos de fabricación de artículos incluso cuando no se tiene algo que decir. De ahí, los artículos no siempre se sitúan en el estado del arte, o al 
menos no lo hacen de manera explícita ni queda claro cómo se insertan, modifican, concretan o analizan dominios de conocimiento reportados en la literatura. Para AIEM, el proceso de llegar a ser más internacional supone garantizar la publicación de artículos con aportaciones nuevas, robustas y precisas a la comunidad de investigación en educación matemática. Dichos artículos deben ser reflejo de una actividad científica e intelectual que rebasa los límites del entorno cercano, que interactúa con y a partir del estado existente del arte, que es lugar de encuentro de conocimientos producidos dentro del área, y que se esfuerza por comunicar cómo el análisis de lo que se sabe enlaza con lo que se obtiene a través de ese análisis. Estos movimientos de AIEM hacia llegar a ser más internacional se han iniciado y, de hecho, están teniendo efectos en el alcance de los movimientos también iniciados hacia llegar a ser más influyente. Todos ellos son fundamentales para que el proyecto de AIEM no se justifique solo por dotar a SEIEM de una revista oficial, sino también por ser un proyecto científico.

\section{Llegar a ser más influyente}

Una revista científica va siendo más internacional - de acuerdo con el significado científico de 'ser internacional' - a medida que los artículos que publica son más útiles e influyentes en la comunidad de investigación de referencia. Otra cuestión es lograr que la revista por sí misma vaya ganando en visibilidad, representatividad, prestigio, reputación e influencia con respecto a dicha comunidad. En la actualidad, la influencia de nuestra revista se puede argumentar de muchos modos. El aumento acelerado en el envío de manuscritos a lo largo del último año es una evidencia en este sentido que, por otra parte, no es ajena a la entrada de AIEM en la base seriada de datos Scopus. También son una evidencia las cifras que proporcionan los instrumentos bibliométricos aplicados a la revista - no a sus artículos individuales - que en el seno del equipo editorial consultamos y que denotan una expansión de la zona de influencia. Otra evidencia similar es la cantidad de visitas al sitio web de la revista, los reenvíos y descargas e incluso los comentarios enviados por correo electrónico o vía repositorios a miembros del equipo. Pero de nuevo, estos focos no son científicos stricto sensu y debe problematizarse el uso sesgado de medidas, indicadores e indizaciones simples como sustitutos disuasorios de una noción científica de influencia.

En el contexto de una revista comprometida con la representación de una comunidad de investigación, el significado de 'ser influyente' radica en el objetivo de marcar y/o abrir tendencias innovadoras en relación con líneas de estudio abiertas y vacíos concretos de conocimiento en la literatura. Al respecto, la consolidación de la política de monográficos anuales es una evidencia del proceso iniciado para el logro de este objetivo. AIEM se publica con regularidad semestral y desde 2017 uno de los números de la revista es monográfico. Esto implica que los números monográficos representan una mitad de la revista. Desde la perspectiva científica, deberá valorarse la importancia de cada uno de estos monográficos especializados en el momento histórico de su publicación y en un periodo posterior de al menos una década. En el momento de su selección - de contenido y de editor invitado -, preparación y publicación, se busca producir un volumen sobre una línea de estudio que compile conocimiento heterogéneamente generado por distintas escuelas teóricas y por tanto por más de un autor y grupo, que pueden no estar de acuerdo sobre cuestiones básicas como cuáles son las direcciones futuras a seguir. Si se hace bien, el número monográfico no deja nunca de ser actual porque se mantiene como una compilación difícilmente exhaustiva pero necesariamente exogámica - del desarrollo de una línea de estudio en un cierto momento histórico. Es aún pronto para realizar un análisis de la influencia de los monográficos de AIEM en la comunidad del área. Ahora bien, 
debemos anticipar su función de innovación investigadora dentro del proyecto AIEM con la selección, preparación y publicación cuidadosa de cada monográfico.

\section{Llegar a ser más formativa}

La perspectiva formativa es fundamental en la consecución de los logros de llegar a ser (más) internacional y de llegar a ser (más) influyente. Esta perspectiva tiene que ver con el proceso de publicación y eventualmente de aprobación de una versión mejorada. En este proceso, tiene un papel clave la terna de actores involucrados autores-evaluadores-editores - con la llamada revisión externa de doble/simple ciego por pares y la revisión última del editor asociado y de la dirección de la revista, ambas sujetas a declaraciones éticas en abierto como signo de seriedad y ecuanimidad. De nuevo, estos métodos de arbitraje y de posterior edición técnica y de estilo, son parámetros que no deben confundirse con el reto de llevar a cabo acciones de apoyo a los autores que sean científicamente formativas en cuanto al contenido de sus escritos. La configuración de un equipo de editores asociados y de una base de evaluadores y de asesores que son académicos profesionales e investigadores expertos en dominios de conocimiento del área de la revista es una condición necesaria. No obstante, el carácter científicamente formativo del proceso de publicación requiere la adopción crítica de objetivos sofisticados relativos a la calidad del contenido. El cumplimiento de estos objetivos no debe darse por supuesto sin acciones orientadas a su logro.

La gran mayoría de los manuscritos recibidos en AIEM pasan por un plazo de tiempo de revisión largo, a menudo con hasta tres rondas y hasta tres revisores que son investigadores expertos en la línea de estudio de los autores. El proceso de publicación se alarga en parte como consecuencia del compromiso del equipo de la revista con hacer de ella un instrumento formativo, de aprendizaje y de desarrollo profesional de investigadores en educación matemática. Cualquier revista científica debe acompañar el proceso indefinido de formación y de desarrollo profesional en investigación del área correspondiente. Así, el significado de 'ser formativa' debe buscarse en cómo se brinda e intercambia conocimiento con los autores de un manuscrito acerca del estado del arte en la línea de estudio de la investigación que se quiere publicar y acerca de cuáles son el impacto potencial y la contribución precisa de dicha investigación a lo que se sabe y a lo que falta por saber en el contexto del estado del arte. Desde esta perspectiva, cuestiones altamente normativizadas como el anonimato del revisor en el arbitraje - que paradójicamente no se acostumbra a mantener en la relación con el editor a pesar de que a este le corresponde argumentar la decisión última - no parecen tan relevantes e incluso pueden resultar contraproducentes. Cuando no se ciega el proceso de revisión, los argumentos proporcionados por los revisores pueden ganar en transparencia, consistencia, rigor y detalle científico en la conversación con los autores. Por esta razón, AIEM es respetuosa con el anonimato de los autores y a la vez flexible ante la práctica creciente de evaluadores que toman la iniciativa de romper el protocolo e incluir su nombre al finalizar sus informes de revisión. No es causal que esta práctica se esté produciendo de manera más global en revistas científicas de una variedad de áreas, a pesar de las directrices marcadas por las principales corporaciones editoriales que siguen recomendando el anonimato doble de autor y de revisor.

\section{Agradecimientos}

A Tomás Sierra, por las conversaciones sobre la función de las revistas científicas en el mundo de la investigación y sobre la función de AIEM en particular. 